\title{
O IMPACTO DA SAÚDE BUCAL NA QUALIDADE DE VIDA EM INDIVÍDUOS COM DRC EM PRÉ-TRANSPLANTE RENAL: ESTUDO PILOTO
}

\author{
The impact of oral health on the quality of life in patients with CKD prior to renal \\ transplantation: a pilot study
}

Aloizio Premoli Maciel ${ }^{1}$, Murilo de Almeida Manoel ${ }^{1}$, Cássia Maria Fischer Rubira ${ }^{1}$, Tricya Nunes Vieira Bueloni ${ }^{2}$, Paulo Sérgio da Silva Santos ${ }^{1}$

\begin{abstract}
RESUMO
O transplante renal é uma importante modalidade de terapia de substituição renal para pacientes portadores de doença renal crônica em estágios avançados. Doenças bucais, como processos infecciosos, inflamação crônica e outros fatores estão fortemente ligadas ao sucesso do transplante. Objetivo: Avaliar o impacto da saúde bucal na qualidade de vida de indivíduos com DRC, no período pré-transplante. Métodos: Foram avaliadas em 25 indivíduos em programação de transplante renal as alterações bucais, sialometria mecanicamente estimulada (SME) e não estimulada (SNE), condição periodontal pelo índice periodontal comunitário, índice gengival e de placa e aplicação do questionário de qualidade de vida OHIP-14. Resultados: O ressecamento labial esteve presente em 14 (56\%) casos, precedido de saburra lingual $10(40 \%)$, língua fissurada $9(36 \%)$, candidíase 7 (28\%), atrofia de papilas linguais $4(16 \%)$, palidez da mucosa $4(16 \%)$ e $2(8 \%)$ casos de abscesso periodontal agudo. A hipossalivação foi constatada em 17 (68\%) pacientes que realizaram SNE e em 13 (52\%) pacientes sob SME. A maioria dos pacientes possuia acúmulo de biofilme bacteriano e inflamação gengival moderada, com sangramento à manipulação e edema, com $82 \%$ de sangramento à sondagem, $88 \%$ com cálculo dentário e $52 \%$ com bolsa periodontal com sondagem entre 4 e $5 \mathrm{~mm}$ de profundidade. Na presente amostra, $32 \%$ dos pacientes tiveram médio impacto da saúde bucal sobre a qualidade de vida. Conclusão: Infecções bacterianas e fúngicas bucais foram achados comuns e podem estar relacionados à hipossalivação. Essas condições provocaram impacto negativo na qualidade de vida dos indivíduos em programação de transplante de rim.
\end{abstract}

Descritores: Insuficiência Renal Crônica; Manifestações Bucais; Qualidade de Vida; Transplante renal.

\footnotetext{
Instituições:

1 Departamento de Cirurgia, Estomatologia, Patologia e Radiologia da Faculdade de Odontologia de Bauru da Universidade de São Paulo, Bauru, SP.

2 Setor de Hemodiálise do Hospital Estadual de Bauru, Bauru, SP.
}

\section{Correspondência:}

Paulo Sérgio da Silva Santos

Al. Dr. Octávio Pinheiro Brisolla, 9-75, CEP 17012-901, Bauru, SP

Tel.: (14) 3226-6113

E-mail: paulosss@fob.usp.br

\section{INTRODUÇÃO}

Doença Renal Crônica (DRC) é um progressivo e irreversível declínio da função renal, associado à redução da taxa de filtração glomerular. ${ }^{1,2}$ Além das manifestações sistêmicas que aparecem associadas à $\mathrm{DRC}$, as alterações metabólicas e patofisiológicas associadas às respectivas modalidades de tratamento podem comprometer a saúde bucal. Não havendo contraindicação, os indivíduos com DRC podem ser submetidos ao transplante renal e considera-se prudente a adequação bucal pré-transplante, principalmente para a prevenção de potenciais infecções sistêmicas de origem bucal, ${ }^{3,4}$ devido à imunossupressão à qual o transplantado será submetido. ${ }^{5}$ 
Em relação às fontes de infecção bucal que podem gerar algum comprometimento no candidato ao transplante, as mais comuns são: cárie dental, doenças pulpares, doenças periodontais, infecções oportunistas fúngicas e virais, como a candidíase oral e infecção pelo vírus do herpes simples ${ }^{6-9}$ ou de manifestações orais relacionadas à hiperuremia como a estomatite urêmica, uma lesão oral associada principalmente à falência renal aguda. ${ }^{10}$ A doença periodontal é considerada a condição bucal mais grave e prevalente encontrada nesses pacientes com DRC ${ }^{3,11,12}$ e que diminuem significativamente a taxa de sobrevida dos pacientes submetidos à hemodiálise, provavelmente, em função dos patógenos periodontais alcançarem a corrente sanguínea, ${ }^{9}$ levando a infecções, desnutrição por perdas energéticas e complicações ateroscleróticas. ${ }^{7,13}$

A hipossalivação é uma condição que acompanha a DRC e é um sinal clínico importante, pois a saliva está diretamente ligada à saúde em geral e à homeostasia dos tecidos bucais, ${ }^{14}$ sendo que a diminuição do fluxo salivar afeta a manutenção dos tecidos moles e duros da boca, ocasionando a diminuição das propriedades de limpeza da saliva, alteração dos processos de cárie e da doença periodontal, deglutição, lubrificação, digestão, na formação de biofilme microbiano em outros processos fisiológicos. ${ }^{15,16}$

Há várias publicações mostrando pesquisas sobre a condição bucal dos indivíduos com DRC, ,2,3,5,-9 mas raros estudos pesquisaram a relação das doenças bucais nesses indivíduos e seu impacto na qualidade de vida. ${ }^{17}$ Diante das alterações bucais que ocorrem em um paciente com DRC e a necessidade de manter uma condição bucal saudável para diminuir essas alterações, o presente estudo piloto teve por objetivo avaliar o impacto da saúde bucal na qualidade de vida de indivíduos com DRC em pré-transplante de rim.

\section{PACIENTES E MÉTODOS}

Este estudo foi caracterizado como uma pesquisa observacional de levantamento epidemiológico, quantitativo e descritivo, com o intuito de verificar a prevalência de alterações bucais de indivíduos com DRC e o impacto da saúde bucal na qualidade de vida.

Este estudo piloto foi composto de 25 indivíduos diagnosticados com DRC em hemodiálise, com idade acima de 18 anos, em fila de transplante de rim que aceitaram participar voluntariamente da pesquisa. Excluímos indivíduos que não apresentaram condição sistêmica de receber avaliação bucal e com doenças infectocontagiosas (ex: Hepatites, AIDS).
Os indivíduos foram submetidos à avaliação clínica odontológica, através de anamnese e exame físico, feitos por um cirurgião-dentista calibrado, registrando informações epidemiológicas como o sexo, idade, tempo de diagnóstico da doença de base, tempo de hemodiálise e doenças associadas. Foram coletados dados sobre a doença de base e tratamento odontológico recente. Foi também realizado o registro de possíveis sintomas correlacionados com a DRC, como ardência bucal, xerostomia, disfagia, disgeusia, avaliação do fluxo salivar mecanicamente estimulado e não estimulado. Foram registradas alterações estomatológicas, condição periodontal (Índice Periodontal Comunitário - IPC, Índice Gengival e de Placa - IG, IP), conforme preconizado pela Organização Mundial de Saúde ${ }^{18} \mathrm{e}$ por Loe e Silness. ${ }^{19-21}$

Foi aplicado o questionário Oral Health Impact Profile (OHIP-14) ${ }^{22-24}$ para avaliar o impacto das condições de saúde bucal sobre a qualidade de vida.

\section{RESULTADOS}

Foram avaliados ${ }^{25}$ pacientes com DRC durante 0 procedimento de hemodiálise em ambiente hospitalar. Desse grupo, 14 (56\%) eram do gênero masculino e 11 (44\%) do feminino, com idade média de 60 anos e mediana de 62 anos, variando entre 37 a 85 anos. Em média, os pacientes possuem 6,16 anos de tempo de diagnóstico da DRC, com 3,34 anos realizando hemodiálise.

Os principais fatores etiológicos da DRC foram a associação de diabetes tipo 2 com hipertensão arterial não controlada, acometendo cerca de 16 (64\%) pacientes. Outros fatores formam a diabetes tipo 2 isolada em quatro (16\%) casos, hiperuremia em dois (8\%) casos e hipertensão arterial isolada, um (4\%) caso de rins policísticos e um (4\%) caso de infecção renal. Em geral, 19 (76\%) pacientes possuíam diabetes mellitus e 16 (64\%) pacientes, hipertensão.

Durante o atendimento odontológico, quatro (16\%) pacientes relataram queixas álgicas bucais, quatro $(16 \%)$ possuíam disfagia, seis (24\%) apresentavam ardor bucal, 11 estavam com (44\%) xerostomia e 11 (44\%) com disgeusia. Foi realizada sialometria mecanicamente estimulada durante cinco minutos, 12 (48\%) pacientes estavam com o fluxo salivar estimulado normal e 13 $(52 \%)$ com o fluxo salivar baixo. Desses pacientes com hipossalivação, nove (36\%) foram classificados com fluxo salivar muito baixo segundo Flink $\mathrm{H}$ et al. $2008,25 \mathrm{com}$ valores inferiores a $0,7 \mathrm{ml} / \mathrm{min}$. A média da sialometria mecanicamente estimulada da amostra foi de $0,74 \mathrm{~mL} / \mathrm{min}$ e mediana de $0,62 \mathrm{~mL} / \mathrm{min}$. 
Em relação à sialometria não estimulada, cerca de oito $(32 \%)$ pacientes foram avaliados com fluxo salivar normal e 17 (68\%) com hipossalivação; destes, 14 (56\%) pacientes foram classificados com fluxo salivar muito baixo, com valores inferiores a $0,1 \mathrm{ml} / \mathrm{min}$. A média da sialometria não estimulada da amostra foi de $0,22 \mathrm{~mL} /$ min e mediana de $0,16 \mathrm{~mL} / \mathrm{min}$.

As principais alterações bucais encontradas foram: ressecamento labial em 14 (56\%) casos,10 (40\%) casos com saburra lingual, nove (36\%) de língua fissurada, sete (28\%) de candidíase, cinco (20\%) de ressecamento bucal, quatro (16\%) casos de despapilamento lingual, quatro (16\%) de palidez da mucosa oral e somente dois (8\%) casos de abscesso periodontal agudo. Nenhum caso de estomatite urêmica foi observado neste estudo.

As avaliações periodontais foram realizadas somente em 17/25 dos pacientes, já que oito eram edêntulos e não se aplicavam a essa avaliação. Dos que foram avaliados pelo índice de placa (17 indivíduos), um $(5,88 \%)$ possuía ausência de biofilme dental, três $(17,66 \%)$ apresentaram biofilme na margem da gengiva marginal livre, sete $(41,17 \%)$ apresentavam acúmulo de biofilme microbiano dentro da bolsa periodontal, sendo possível ser observada visualmente na margem gengival do dente ao exame clínico e, por fim, seis $(35,29 \%)$ possuíam biofilme dentro de bolsa periodontal com visualização clínica na margem gengival do dente e em outras localidades da coroa dentária.

$O$ índice gengival também foi aplicado nos mesmo pacientes avaliados pelo índice de placa. Dos pacientes avaliados, apenas um $(5,88 \%)$ apresentou gengivas com aspectos clínicos normais, quatro (23,52\%) possuíam gengivas sutis, alterações de coloração e volume sem sangramento à palpação, cinco $(29,41 \%)$ apresentaram inflamação gengival moderada com vermelhidão, edema, de aspecto brilhante e sangrentos à manipulação e, finalmente, sete $(41,17 \%)$ pacientes estavam com inflamação gengival severa, caracterizada por vermelhidão com ulcerações ou com tendências a sangramento espontâneo.

Através do índice periodontal comunitário (IPC), foram constatadas alterações periodontais em todos os 17 pacientes avaliados, com 14 (82,35\%) casos apresentando sangramento gengival à sondagem, $15(88,23 \%)$ casos com cálculo dentário extra e intra sulco gengival, nove $(52,94 \%)$ casos com bolsa de 4 a $5 \mathrm{~mm}$ de profundidade de sondagem e um $(5,88 \%)$ caso com bolsa periodontal. Em relação à amostra total, esses percentuais caíram para $56 \%, 60 \%, 36 \%$ e $4 \%$, respectivamente.
A aplicação do questionário de vida OHIP-14 mostrou que oito $(32 \%)$ pacientes avaliados possuíam um impacto médio na qualidade de vida, diminuído por sua condição bucal presente.

Os demais 17 (68\%) indivíduos avaliados possuíam um fraco impacto na qualidade de vida relacionado com a saúde bucal. $\mathrm{Na}$ avaliação das dimensões, encontrouse impacto médio em dor física $(1,364)$ e desconforto psicológico (1,384). Na dimensão de incapacidade psicológica houve impacto fraco $(1,241)$, porém mais expressivo que as demais.

\section{DISCUSSÃO}

Para determinar a hipótese de que alterações bucais específicas possam impactar na qualidade de vida de pacientes com DRC pré-transplante de rim, é necessário saber se tais alterações estão presentes na população estudada, comparando a outros estudos publicados.

Lesões bucais foram constatadas em 11/25 (44\%) indivíduos deste estudo, sendo a candidíase a manifestação mais prevalente, presente em sete (28\%) indivíduos da amostra. Alterações bucais decorrentes da DRC acometeram 20 (80\%) pacientes, e apenas um (4\%) paciente da amostra possuía plena saúde bucal. Outros estudos demonstram valores menores, entre $16,7^{8}$ e $17 \%{ }^{26}$ em relação às lesões bucais, sendo a candidíase também a mais prevalente com $17,2 \%{ }^{27}$ e $5,7 \%^{8}$ das doenças observadas.

Apesar de não se saber ao certo quais mecanismos estão envolvidos no acúmulo de biofilme bacteriano e na formação de cálculos dentários nos pacientes com DRC em hemodiálise, sabe-se que, pelo menos esse grupo de pacientes possui maiores formações de cálculo dentário do que seus pares sem a DRC. ${ }^{28} \mathrm{O}$ acúmulo de biofilme e cálculo dentário é significativamente alto em pacientes em hemodiálise, principalmente quando comparados a indivíduos saudáveis. ${ }^{26}$ Neste estudo, os indivíduos com DRC, em sua maioria, apresentaram acúmulo moderado de biofilme bacteriano dentro de bolsa periodontal, fator este agressor aos tecidos gengivais, provocando sangramento e, consequentemente, a formação de bolsas periodontais.

$\mathrm{Na}$ amostra de 25 indivíduos, 15 (60\%) apresentavam acúmulo de cálculo dentário, 14 (56\%) possuíam sangramento gengival positivo, com índice gengival indicando inflamação gengival moderada com tendências a edema, vermelhidão e sangramento espontâneo ou à apalpação, em 58\% dos pacientes. Quando comparada a outros estudos, a doença periodontal permanece alta, 
visto que os valores encontrados foram de $29 \%{ }^{29}$ e $12 \% .^{8}$

Quando avaliada a presença de bolsa periodontal em pacientes com DRC, encontrou-se a presença de bolsa maior que $4 \mathrm{~mm}$ em 14 (8,8\%) indivíduos. ${ }^{29}$ No presente estudo, foram encontrados índices bem mais elevados (36\%) nos pacientes que possuíam bolsa periodontal maior que $4 \mathrm{~mm}$ e um (4\%) maior que $6 \mathrm{~mm}$, caracterizando a persistência da doença periodontal como um foco de infecção. Um fator que colabora para os altos índices de sangramento gengival e profundidade de sondagem periodontal, é a presença de diabetes mellitus em 19/25 (76\%) dos indivíduos com DRC avaliados neste estudo, o que, juntamente com a maior formação de biofilme microbiano e cálculo dentário ocorridos nesses indivíduos, contribuem para o avanço da profundidade da bolsa mais rapidamente e, consequentemente, das complicações advindas da doença periodontal como reabsorção óssea, exposição radicular ao meio bucal e a perda dentária.

A disgeusia foi o sintoma prevalente em $31,7 \%{ }^{30}$ e $45,5 \%{ }^{27}$ dos indivíduos com DRC avaliados, descritos na literatura. Um sintoma menos relatado é o ardor bucal, constatado em $13,1 \%{ }^{27}$ e $18 \%{ }^{26}$ dos pacientes com DRC em hemodiálise. Neste estudo, encontrou-se a queixa de disgeusia em 11 (44\%) pacientes e o sintoma de ardor bucal em seis (24\%).

Em pacientes sob hemodiálise, no Brasil, foi constatada xerostomia em $40 \%$ dos indivíduos avaliados, ${ }^{29}$ o que corrobora com os 11 (44\%) encontrados neste presente estudo. Um estudo realizado em população mexicana obteve valor similar, com $44 \%$ dos pacientes com xerostomia ${ }^{27}$ e em outros estudos, os valores foram de $31,9 \%,{ }^{30} 30,1 \%{ }^{31}$ e $12 \% .{ }^{8}$ A xerostomia é um sintoma que pode indicar a presença de ressecamento bucal ou hipossalivação, porém para a constatação da hipossalivação é necessária a mensuração da quantidade presente de saliva na boca, através da sialometria.

Indivíduos com DRC, quando comparados a um grupo controle de indivíduos normoreativos, determinou-se relação entre a DRC e a hipossalivação, através de sialometria não estimulada, com valores entre 0,30 e $0,18 \mathrm{ml} / \mathrm{min}$ nos indivíduos com DRC, estatisticamente significativa. ${ }^{30,32}$ Outras pesquisas observaram valores que variaram entre zero e $0,5 \mathrm{~mL} / \mathrm{min} 26,29,33$ para sialometria não estimulada, e entre zero e $2,5 \mathrm{~mL} / \mathrm{min}$ $29,33,34$ para a sialometria mecanicamente estimulada. No presente estudo, a sialometria não estimulada obteve valores entre 0,02 e $0,8 \mathrm{~mL} / \mathrm{min}$, com mediana de $0,16 \mathrm{~mL} / \mathrm{min}$, e a mecanicamente estimulada entre 0,04 e $2,04 \mathrm{~mL} / \mathrm{min}$ com mediana de $0,62 \mathrm{~mL} / \mathrm{min}$. Os valores da mediana de ambas as sialometrias são compatíveis para hipossalivação, ${ }^{25}$ o que indica que tal alteração bucal é presente na maioria dos pacientes avaliados com DRC pré-transplante de rim.

A hipossalivação avaliada por sialometria estimulada mecanicamente foi encontrada em $19 \%$ dos pacientes sem DRC. ${ }^{35}$ No presente estudo, $13(52 \%)$ pacientes com DRC foram diagnosticados com hipossalivação através da análise da sialometria estimulada e 17 (68\%) através da sialometria não estimulada.

O ressecamento bucal presente em pacientes com DRC foi associado à combinação entre a restrição de volume existente e o aumento nos níveis salivares de ureia ligados a essa condição, ${ }^{28}$ podendo haver possíveis danos às glândulas submandibulares durante períodos de hiperuremia ${ }^{36}$

Existem sinais representativos de ressecamento das mucosas bucais que podem ser encontrados na boca; um deles é o despapilamento das papilas filiformes da língua, o qual deixa a língua com aspecto liso, brilhante e eritematoso. No presente estudo, o ressecamento da mucosa bucal foi constatado em cinco $(20 \%)$ pacientes avaliados, resultado inferior ao de outro estudo em que essa porcentagem foi de $74,2 \% .{ }^{26} \mathrm{O}$ despapilamento lingual foi constatado em quatro (16\%) pacientes deste presente estudo, similar ao encontrado em outros estudos em proporções menores de $1,7 \%{ }^{8}$

A principal alteração bucal encontrada foi ressecamento labial e saburra lingual, acometendo 14 (56\%) e 10 (40\%) pacientes, respectivamente. Essas alterações bucais também foram as mais prevalentes, com $28,3 \%$ para ressecamento labial e 18,2\% para saburra lingual. ${ }^{27}$

A hipossalivação em indivíduos sem a DRC é condição suficiente para impactar como condição bucal na qualidade de vida, promovendo-a através de interrupções alimentares, dificuldade em relaxar durante as atividades diárias e a sensação de piora de vida ${ }^{35}$

No presente estudo, 11 (44\%) participantes possuíam xerostomia, 13 (52\%) possuíam hipossalivação durante a sialometria estimulada e 16 (68\%) durante a sialometria não estimulada. Através da avaliação geral do OHIP-14, o impacto na qualidade de vida relacionado à saúde bucal foi moderado em oito (32\%) pacientes avaliados. O impacto negativo na qualidade de vida desses indivíduos pode ser atribuído a altos índices de xerostomia (44\%) e hipossalivação (68\%), os quais consequentemente podem ter influenciado na piora da dimensão de desconforto psicológico em relação às demais dimensões avaliadas pelo OHIP-14. 
Sabe-se que a melhora da qualidade de vida em pacientes com DRC está relacionada à realização do transplante de rim. ${ }^{37}$

O diagnóstico e tratamento de comorbidades, como infecções orais odontogênicas ou oportunistas, no período pré-transplante de rim, contribuem para o aumento da sobrevida do órgão doado. ${ }^{38}$

Em uma revisão integrativa abordando diversos artigos, foi encontrado que diagnóstico e tratamento das manifestações bucais em pacientes com DRC antes e após o transplante de rim são relevantes para a manutenção do órgão transplantado, e são eles a orientação de higiene oral, confecção de restaurações dentárias, tratamento periodontal, aplicação de antifúngicos, quando presentes, como a nistatina e o fluconazol e extrações dentárias, que são as terapêuticas mais utilizadas para a adequação oral desses indivíduos. ${ }^{38}$

\section{CONCLUSÃO}

Indivíduos com DRC e candidatos ao transplante de rim apresentam com frequência xerostomia e alterações bucais, como hipossalivação, ressecamento bucal e labial, saburra lingual e candidíase oral, o que contribui para impactar negativamente a qualidade de vida.

\section{ABSTRACT}

Introduction: Renal transplantation is an important modality of renal replacement therapy for patients with chronic kidney disease in advanced stages. Unlike oral diseases as foci of infection, chronic inflammation and other factors are directly linked to a successful transplant. The purpose of this research is to assess the impact of oral health on the quality of life of CKD-bearer individuals in the pre-transplantation period. Methods: It was assessed 25 individuals in kidney transplantation schedule in relation to oral alterations, mechanically stimulated sialometry (MSS) and nonstimulated sialometry (NSS), periodontal status by community periodontal index, gingival index, plaque index, and the OHIP-14 quality of life questionnaire was applied. Results: Lip dryness was present in $14(56 \%)$ cases, preceded by coated tongue $10(40 \%)$, fissured tongue $9(36 \%)$, candidiasis $7(28 \%)$, lingual papilla atrophy $4(16 \%)$, pallor of the buccal mucosa $4(16 \%)$ and $2(8 \%)$ cases of acute periodontal abscess. Hyposalivation was observed in $17(68 \%)$ patients who underwent SNE, and in 13 (52\%) patients under SME. Most patients have microbial biofilm accumulation inside the periodontal pocket, and moderate gingival inflammation with bleeding under manipulation and edema, with $82 \%$ bleeding probing, $88 \%$ with dental calculus and $52 \%$ with periodontal pocket under probing between 4 and 5 $\mathrm{mm}$ depth. In the present sample, $32 \%$ of patients had medium impact of oral health on the quality of life. Conclusion: Mouth bacterial and fungal infections were common and may be related to hyposalivation. Those conditions had a negative impact on the quality of life in individuals in kidney transplantation schedule.

Keywords: Chronic Kidney Disease; Oral Manifestations; Quality of Life; Kidney Transplantation. 


\section{REFERÊNCIAS}

1- Chan CM James, Williams DM, Roth KS. Kidney failure in infants and children.Pediatr Rev. 2002;23(2):47-60.

2- Proctor R, Kumar N, Stein A, Moles D, Porter S. Oral and Dental Aspects of Chronic Renal Failure. J Dent Res. 2005;84(3):199-208.

3- Cengiz MI, Sümer P, Cengiz S, Yavuz U. The effect of duration of the dialysis in hemodialysis patients on dental and periodontal findings.Oral Disease. 2009;15(5):336-41.

4- Ertugrul F, Elbek-Cubukcu C, Sabah E, Mir S. The oral health status of children undergoing hemodialysis treatment. Turk J Pediatr. 2003;45:108-13.

5- Guggenheimer J, Eghtesad B, Stock DJ. Dental management of the (solid) organ transplant patient.OralSurg Oral Med Oral Pathol Oral RadiolEndod. 2003;95(4):383-9.

6- Ahmadieh A, Baharvand M, Fallah F, Djaladat H, Eslani M. Oral microflora in patients on hemodialysis and kidney transplant recipients. Iran J KidneyDis. 2010;4(3):227-31.

7- Akar H, Akar GC, Carrero JJ, Stenvinkel P, Lindholm B. Systemic consequences of poor oral health in chronic kidney disease patients. Clin J Am SocNephrol. 2011;6(1):218-26

8- Oyetola EO, Owotade FJ, Agbelusi GA, Fatusi OA, Sanusi AA. Oral findings in chronic kidney disease: implications for management in developing countries. BMC Oral Health.2015;15(1):24.

9- de la Rosa-García E, Mondragon-Padilla A, IrigonyenGnacho M, BustamanteRM. Oral lesions in a group of Kidney transplant patients. Med Oral Patol Oral Cir Bucal. 2005;10(3):196-204.

10- Jaspers MT Unusual oral lesions in an uremic patient. Review of literature and report of a case. Oral Surg Oral Med Oral Pathol Oral RadiolEndod. 1975;39(6):934-44.

11- Chambrone L, Foz AM, Guglielmetti MR, Pannuti CM, Artese HP, Feres M, et al.. Periodontitis and chronic kidney disease: a systematic review of the association of diseases and the effect of periodontal treatment on estimated glomerular filtration rate. J ClinPeridontol. 2013; 40:443-56.

12- Sharma P, Dietrich T, Ferro CJ, Cockwell P, Chapple IL. Association between periodontitis and mortality in stages 3-5 chronic kidney disease: NHANES III and linked mortality study. J ClinPeriodontol. 2016;43(2):104-13.

13- Borawski J, Wilczyńska-Borawska M, Stowska W, Myśliwiec M. The periodontal status of pre-dialysis chronic kidney disease and maintenance dialysis patients.Nephrol Dial Transplant. 2007;22(2):457-64.

14- Dawes C, Pedersen AM, Villa A, Ekström J, Proctor GB, Vissink $A$, et al. The functions of human saliva: $A$ review sponsored by the World Workshop on Oral Medicine VI. Arch Oral Biol. 2015;60(6):863-74.
15- Edgar WM, O'Mullane DM. Saliva and oral Health. 2nd ed. London: British Dental Journal; 1996.

16- Tarzia O. Halitose: um desafio que tem cura. 2nd ed. Rio de Janeiro: EPUB; 2003.

17- Guzeldemir E, Toygar H, Tasdelen B. Oral healthrelated quality of life and periodontal health status in patients undergoing hemodialysis. J Am Dent Assoc. 2009;140(10):1283-93.

18- World Health Organization (WHO). Oral Health Surveys Basic Methofd.Oms. 2013;1.137.

19- Löe $H$. The gingival index, the plaque index and the retention index systems. J Periodontol. 1967;38(6):610-6.

20- Löe H, Silness J. Periodontal disease in pregnancy. I. Prevalence and severity. ActaOdont Scand. 1963;21(6):533-51.

21- Silness J, Löe H. Periodontal disease in pregnancy II. correlation between oral hygiene and periodontal conditions. ActaOdontScand. 1964;22(1):121-35.

22- Bastos RS, Carvalho SE, Xavier A, Caldana LM, Bastos JRM, Lauris JRP. Dental caries related to quality of life in two Brazillian adolescent grups: a cross-sectional randomised study. IntDent J. 2012;62(3):137-43.

23- Oliveira BH, Nadanovsky P. Psychometric properties of the Brazilian version of the Oral Health Impact Profile-short form. Community Dent Oral Epidemiol. 2005; 33:307-14.

24- Slade GD, Strauss, RP, Atchison KA, Kressin NR, Locker $D$, Reisine ST. Assessing oral health outcomes-measuring health status and quality of life. Community Dent Health. 1998;15:3-7.

25- Flink H, Bergdahl M, Tegelberg Å, Rosenblad A, Lagerlöf F. Prevalence of hyposalivation in relation to general health, body mass index and remaining teeth in different age groups of adults. Community Dent Oral Epidemiol. 2008;36(6):523-31.

26-Gavalda C, Bagán JV, Scully C, Silvestre FJ, Milián MA, Jimenez Y. Renal hemodialysis patients: oral, salivary, dental and periodontal findings in 105 adult cases. Oral Diseas. 1999;5(4),299-302.

27- de la Rosa-García E, Mondragón-Padilla A, Aranda-Romo $\mathrm{S}$, Bustamante-Ramírez MA. Oral mucosa symptoms, signs and lesions, in end stage renal disease and non-end stage renal disease diabetic patients. Med Oral Patol Oral Cir Bucal. 2006;11(5):271.

28- Epstein SR, Irwin M, Irwin W. Scopp.Salivary composition and calculus formation in patients undergoing hemodialysis.JPeriodont. 1980;51(6):336-8.

29- Cunha FL, Tagliaferro EP, Pereira AC, MeneghimMC,HeblingE.Oral health of a brazilian population on renal dialysis. Spec Care Dentist.2007;27(6):227-31. 
30- Kho HS, Lee SW, Chung SC, Kim YK. Oral manifestations and salivary flow rate, $\mathrm{pH}$, and buffer capacity in patients with end-stage renal disease undergoing hemodialysis.OralSurg Oral Med Oral Pathol Oral RadiolEndod.1999;88(3):316-9.

31- Bots CP, Brand HS, Veerman EC, Valentijn-Benz M, van Amerongen BM, Valentijn RM, etal.Interdialytic weight gain in patients on hemodialysis is associated with dry mouth and thirst. Kidney Int. 2004;66(4):1662-8.

32- Tomás I, Marinho JS, Limeres J, Santos MJ, Araújo J, Diz $P$. Changes in salivary composition in patients with renal failure. Arch Oral Biol. 2008;53(6):528-32.

33- Eresson C, Thorman R, Rodhe Y, MöllerL, Hylander B. DNA damage in salivary gland tissue in patients with chronic kidney disease, measured by the comet assay. Oral Surg Oral Med Oral Pathol Oral RadiolEndod. 2011;112(2),209-15.

34- Martins C, Siqueira WL, Oliveira E, Primo LSD, Nicolau J. Salivary analysis of patients with chronic renal failure undergoing hemodialysis. SpecCare Dentist. 2006;26(5):205-8.
35- Ikebe K, Matsuda KI, Morii K, Wada M, Hazeyama T, Nokubi T, et al. Impact of dry mouth and hyposalivation on oral health-related quality of life of elderly Japanese. Oral Surg Oral Med Oral Pathol Oral RadiolEndod. 2007;103(2):216-22.

36- Rothstein D, Yudis M, Shaw AS, Onesti G. Massive neck swelling secondary to uremic submaxillary gland involvement. Oral Surg Oral Med Oral Pathol Oral Radiol. 1969;27(3):333-6.

37- Álvarez-Rangel LE, Cruz-Santiago J, Meza-Jiménez G, Bernáldez-Gómez G, Ledesma-González VM, CamachoHernández $F$, et al. Modification of Health-related quality of life in kidneytransplant recipients. RevMedlnstMex Seguro Soc. 2015;53:66-9

38- Ikuta CRS, Quispe RA, Premoli A, Rubira CMF, Santos PSS. A equipe multidisciplinar e a ação do cirurgião dentista nos pacientes transplantados renais: uma revisão integrativa. RBM. 2016;73:36-2. 